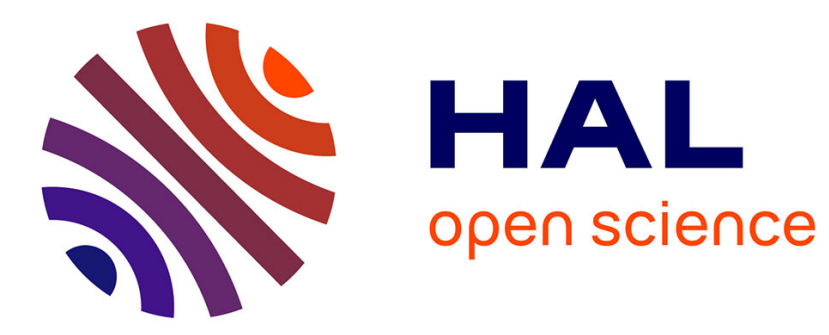

\title{
Adaptive Task-Space Force Control for Humanoid-to-Human Assistance
}

Anastasia Bolotnikova, Sébastien Courtois, Abderrahmane Kheddar

\section{To cite this version:}

Anastasia Bolotnikova, Sébastien Courtois, Abderrahmane Kheddar. Adaptive Task-Space Force Control for Humanoid-to-Human Assistance. IEEE Robotics and Automation Letters, 2021, 6 (3), pp.5705-5712. 10.1109/LRA.2021.3084889 . hal-03170625v3

\section{HAL Id: hal-03170625 \\ https://hal.science/hal-03170625v3}

Submitted on 26 May 2021

HAL is a multi-disciplinary open access archive for the deposit and dissemination of scientific research documents, whether they are published or not. The documents may come from teaching and research institutions in France or abroad, or from public or private research centers.
L'archive ouverte pluridisciplinaire HAL, est destinée au dépôt et à la diffusion de documents scientifiques de niveau recherche, publiés ou non, émanant des établissements d'enseignement et de recherche français ou étrangers, des laboratoires publics ou privés. 


\title{
Adaptive Task-Space Force Control for Humanoid-to-Human Assistance
}

\author{
Anastasia Bolotnikova, Sébastien Courtois, and Abderrahmane Kheddar
}

\begin{abstract}
We envision a humanoid robot to serve as a source of additional motion-support forces in assistance for frail persons. In this context, we present a control strategy for a humanoid to adaptively regulate its assistive force contribution. First, we identify a human model torque control for optimal execution of a priori known motion task from sample recordings of this task performed by a healthy individual. We utilize the identified model in the proposed position discrepancy based observer of the human torque contribution, the unknown and unmeasurable variable. We propose an experience-based human contribution model learning strategy that allows to improve the human contribution estimate from trial-to-trial. The target assistive torque contribution is then calculated as the difference between the optimal torque required for the motion task and the estimated human contribution. The target assistive torque is integrated into a multi-robot quadratic programming task-space controller to compute the desired interaction force required for the robot to supply the necessary assistive torque for the human model. We use the non-optimal recordings of the human motion task to emulate human frailty and apply our adaptive force control strategy to demonstrate the results of a humanoid successfully assisting the simulated human model to restore the optimal motion task performance.
\end{abstract}

Index Terms-Physical Human-Robot Interaction, Physically Assistive Devices, Force Control, Motion Control

\section{INTRODUCTION}

D AILY assistance for frail persons and elderly is one of the promising application areas of humanoid robots, e.g. [1], [2], [3]. Humanoids could be specifically designed to be userfriendly, multi-functional and safe [4]. These properties allow us to envision a humanoid providing companionship through social assistance [5] and helping people in need to perform daily chores. Being a platform capable of physical interaction, one of the useful functionalities for humanoids would be to assist frail people with motion tasks that typically require assistance from a human caregiver. Enabling a humanoid to provide such assistance safely and efficiently can help to increase frail person autonomy.

During physical assistance, two sources of force contribute to the human motion: (i) forces that are generated by the human, and (ii) the assistive forces supplied by the robot (Fig. 11). The control challenge in such a scenario lies in that

Manuscript received: December, 21, 2020; Revised February, 22, 2021; Accepted May, 11, 2021.

This paper was recommended for publication by Editor Gentiane Venture upon evaluation of the Associate Editor and Reviewers' comments.

A. Bolotnikova is with École Polytechnique Fédérale de Lausanne, Switzerland anastasia.bolotnikova@epfl.ch

S. Courtois is with SoftBank Robotics Europe, Paris, France

A. Kheddar is with the CNRS-AIST Joint Robotics Laboratory (JRL), Japan and with the CNRS-University of Montpellier, Laboratoire d'Informatique de Robotique et de Microélectronique de Montpellier (LIRMM), France

Digital Object Identifier (DOI): see top of this page.

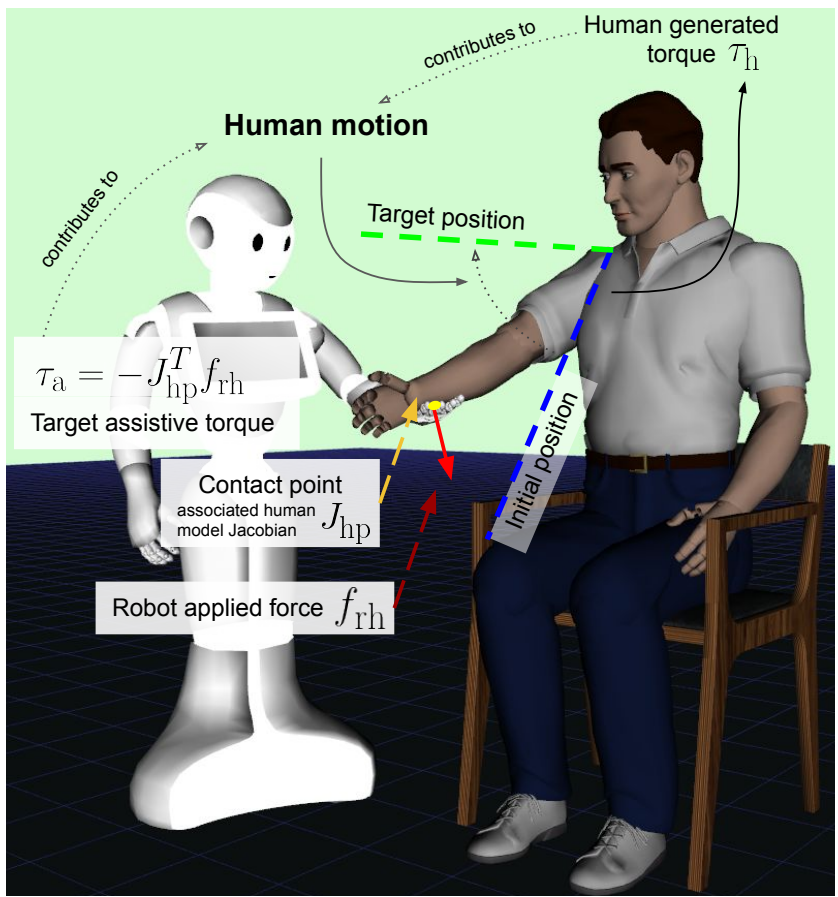

Fig. 1: Humanoid-to-human physical assistance in motion.

human force contribution is not known to the robot and cannot be directly measured. The exact intended human motion is not known either. Hence, the assisted human motion can be performed correctly only if the robot force contribution to the task is adaptively regulated to account for the presence of unknown and unmeasurable human contribution. Indeed, lower force than required wouldn't provide the necessary assistance; more force than required could engender fear, stress or deviate the motion greatly from the way it should be correctly executed, causing discomfort and potentially harm to the human.

Our contributions are as follows. First, as an extension to the multi-robot task-space quadratic programming controller (MQP) framework [6], we integrate a human model as an additional 'robot'. Indeed, in the original work presenting the MQP [6], this extension was left as future work that we partly address in this letter. By doing so, constraints inherent to frailty such as human reduced range of motion, muscles torques limits, absence of limb... are integrated straightforwardly to be accounted for during robot control. Second, based on this framework, we propose a control scheme to provide the strictly necessary assistive force for a priori known human motion task while accounting for possible human force contribution to the task. By using knowledge on the human expected motion for 
a given task performed without muscle strength limitations, an associate model of human torque control is identified (Sec. III-A). This model is used in a position discrepancy based human torque contribution observer (Sec. III-B). An experience-based prediction strategy allows to improve the human contribution estimate from trial-to-trial (Sec. III-C). The assistive torque required to support a motion task is then defined as a difference between the torque required for the correct motion execution and the estimate of the human contribution. Finally, the target assistive torque is integrated into the MQP to compute (and servo on) the desired force the robot applies to provide the assistive torque (Sec. III-D). We use sample recordings of non-optimal human movement to emulate human frailty and demonstrate how our method enables the humanoid-to-human assitance (Sec. IV). To the best of the authors' knowledge, this work is the first to devise task-space optimal control -extensively used in multilimbs redundant robots, to direct humanoid-human physical interaction to assist frail human motion.

\section{Problem Statement}

For a common and well-defined human task (e.g. target reaching [7], [8], sit-to-stand transfer [9]...) human motion prediction can be obtained from theory computational models (simulation), or from sample recording of the motion executed correctly unassisted by individuals using motion capture systems. Human motion prediction is a very active research topic in itself, e.g. [10], [11]. Such knowledge can be exploited and used in our context; however, it needs to be filtered in a one-run execution under the frailty constraints implemented in the MQP (e.g. human with reduced joint limits), which could induce a slightly different motion even in the availability of full muscle strength (no human torque limitation). The MQP task would implement a simple tracking of this ideal taskmotion under all strict integrity-constraints. At the end, for a given task, we collect a time series of the human joint angles $q_{\mathrm{h}}^{\text {task }}(t), t=0, \ldots, T$, where $T$ is the time when the target joint position $q_{\mathrm{h}}^{*}$ for the given task is reached, together with respective joint velocity $\dot{q}_{\mathrm{h}}^{\text {task }}(t)$ and acceleration $\ddot{q}_{\mathrm{h}}^{\text {task }}(t)$. The nominal torque $\tau^{\text {task }}(t)$ required to perform the task motion can be obtained using a sample personalized model of a human body (fixed contacts, no floating base) with dynamic link properties and inverse dynamics (eq. 11.

$$
\tau^{\text {task }}(t)=M_{\mathrm{h}}\left(q_{\mathrm{h}}^{\text {task }}(t)\right) \ddot{q}_{\mathrm{h}}^{\text {task }}(t)+C_{\mathrm{h}}\left(q_{\mathrm{h}}^{\text {task }}(t), \dot{q}_{\mathrm{h}}^{\text {task }}(t)\right)
$$

where $M_{\mathrm{h}}$ is a human model inertia matrix and vector $C_{\mathrm{h}}$ incorporates Coriolis, centrifugal and gravity terms. There are reliable methods to identify inertia parameters of healthy [12], [13] or frail [14] persons.

In the physical assistance process, the total torque required to perform a task all along its motion is the sum of what the human can possibly generate as torque $\tau_{\mathrm{h}}$, and the assistive torque $\tau_{\mathrm{a}}$ provided by the robot by means of multi-contact physical interaction, i.e. by applying forces from robot to human $f_{\mathrm{rh}}$ at multiple points $\mathrm{p}$ (in practice, one or two) located on a human (eq. 2).

$$
\tau^{\text {task }}=\tau_{\mathrm{h}}+\tau_{\mathrm{a}}=\tau_{\mathrm{h}}+J_{\mathrm{hp}}^{T} f_{\mathrm{hr}}
$$

where $J_{\text {hp }}$ is a human body model stacked Jacobians that maps the forces $\left(f_{\mathrm{hr}}=-f_{\mathrm{rh}}\right)$, applied at possibly multiple points $\mathrm{p}$, into human model joint torques.

For a frail human $\tau_{\mathrm{h}}<\tau^{\text {task }}$, i.e. human muscular strength is not sufficient for achieving the task motion correctly. The goal of the robotic assistance is to apply the contact forces that supplement the human-generated torques such that the motion resembles as closely as possible the expected one. Thus, roughly speaking, the amount of the required assistive torque is the difference between the total torque required to perform the task and the human generated torque (eq. 3 .

$$
\tau_{\mathrm{a}}=\tau^{\mathrm{task}}-\tau_{\mathrm{h}}
$$

The human muscle generated torque is not known to the robot a priori and cannot be directly measured. The main challenge in the assistance process is the adaptation of the robot force contribution to the unknown and unmeasurable variable $\tau_{\mathrm{h}}$. In this letter, we suggest that the robot contribution adaptation is achieved by observing the discrepancies between expected and measured human motion. This is because the latter can be estimated from vision, see e.g. [15].

In the following, we present the position discrepancy based human torque observer coupled with an experience-based prediction model of $\tau_{\mathrm{h}}$. Then, we integrate the target assistive torque into the MQP.

\section{PROPOSED METHOD}

\section{A. Identifying reference task torque control model}

Once we identify the task torque trajectory $\tau^{\text {task }}$ (eq. 1 , the relation between task error $e$ (as defined in [6]) evolution data and the total torque required for the task to be performed can be identified. In this study, we use a neural network (NN) model for identifying this relation (eq. 4).

$$
\tau^{\mathrm{task}}=\mathrm{NN}\left(e, \int e, \dot{e}\right)
$$

Given the current task error state $\left(e, \int e, \dot{e}\right)$, this model predicts what would be the total torque if a given human subject (represented by a personalized human model) would execute the task-motion unassisted $\left(\tau_{\mathrm{a}}=0\right)$. The network structure in our study comprises 3 neurons in the input layer, 12 neurons in a single hidden layer and a single neuron in the output layer. Based on our experimentations with the model fitting, such a structure proved to be an optimal compromise between model complexity and prediction accuracy.

Next, we show what role $\tau^{\text {task }}$ plays in the estimation of $\tau_{\mathrm{h}}$ and subsequent computation of the target assistive torque $\tau_{\mathrm{a}}$. In the Sec. IV we demonstrate the use of eq. (4) for $\tau^{\text {task }}$ computation in the proposed control framework (Fig. 2).

\section{B. Human torque contribution observer}

We derive a position discrepancy based observer for estimating the human torque contribution $\tau_{\mathrm{h}}$ to the task. At the very start of the interaction, we use an initial guess of the human torque contribution estimate, $\hat{\tau}_{\mathrm{h}}^{\text {init }}$, to compute the target assistive torque $\tau_{\mathrm{a}}^{*}=\tau^{\text {task }}-\hat{\tau}_{\mathrm{h}}^{\text {init }}$. 


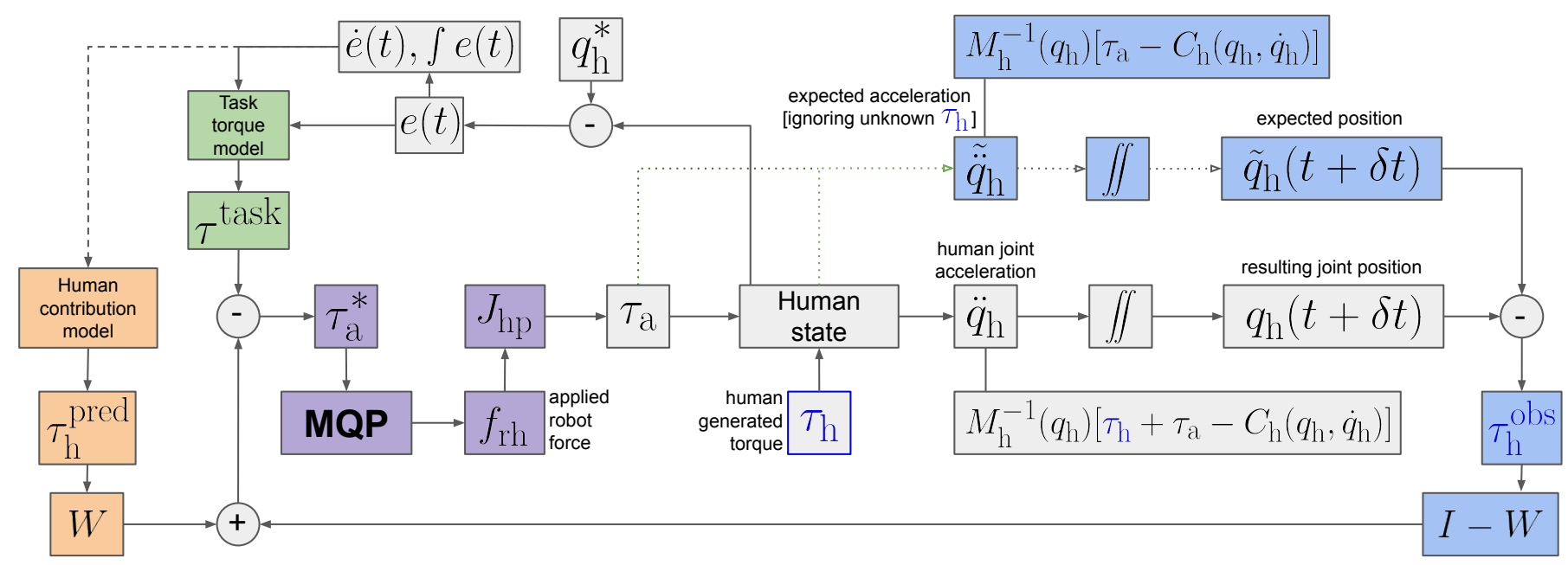

Fig. 2: The proposed control scheme for adaptive humanoid-to-human assistance with a motion task.

As shown in Fig. 22 the target assistive torque is passed to the MQP block. Indeed, the MQP computes the desired robot motion together with consistent forces for the robot to apply at contact points so as to generate $\tau_{\mathrm{a}}^{*}$ in the human model joints, through physical interaction. More details on the use of the human model in the MQP controller are provided later in Sec. III-D Such MQP based control of the humanoid results in $f_{\mathrm{rh}}$ forces being applied on the human body. These forces are mapped into human joint assistive torques, $\tau_{\mathrm{a}}$, via stacked contact Jacobian, $J_{\text {hp }}$, (eq. 2).

In order to observe yet unknown human contribution to the task $\tau_{\mathrm{h}}$, we assume $\tau_{\mathrm{a}}$ to be the only joint torque acting on the human model. With this assumption in mind, we compute expected acceleration of the human model joints via forward dynamics (FD, eq. 5].

$$
\tilde{\ddot{q}}_{\mathrm{h}}=M_{\mathrm{h}}^{-1}\left(q_{\mathrm{h}}\right)\left[\tau_{\mathrm{a}}-C_{\mathrm{h}}\left(q_{\mathrm{h}}, \dot{q}_{\mathrm{h}}\right)\right]
$$

Then, we compute respective expected velocity and position for the following time step $t+\delta t$ (eq. 677)

$$
\begin{aligned}
& \tilde{\dot{q}}_{\mathrm{h}}(t+\delta t)=\dot{q}_{\mathrm{h}}(t)+M_{\mathrm{h}}^{-1}\left(q_{\mathrm{h}}\right)\left[\tau_{\mathrm{a}}-C_{\mathrm{h}}\left(q_{\mathrm{h}}, \dot{q}_{\mathrm{h}}\right)\right] \delta t \\
& \tilde{q}_{\mathrm{h}}(t+\delta t)=q_{\mathrm{h}}(t)+\tilde{\dot{q}}_{\mathrm{h}}(t+\delta t) \delta t= \\
& =q_{\mathrm{h}}(t)+\dot{q}_{\mathrm{h}}(t) \delta t+M_{\mathrm{h}}^{-1}\left(q_{\mathrm{h}}\right)\left[\tau_{\mathrm{a}}-C_{\mathrm{h}}\left(q_{\mathrm{h}}, \dot{q}_{\mathrm{h}}\right)\right] \delta t^{2}
\end{aligned}
$$

After $\tau_{\mathrm{h}}$ and $\tau_{\mathrm{a}}$ are applied to the human model at time $t$, the resulting human joint position $q_{\mathrm{h}}(t+\delta t)$ is estimated (e.g. via any robot embedded motion tracking). This measured quantity can be expressed through integration of forward dynamics, this time taking both sources of torque, $\tau_{\mathrm{a}}$ and $\tau_{\mathrm{h}}$, into account (eq. 8)

$$
\begin{aligned}
& q_{\mathrm{h}}(t+\delta t)=q_{\mathrm{h}}(t)+\dot{q}_{\mathrm{h}}(t) \delta t+ \\
& \quad+M_{\mathrm{h}}^{-1}\left(q_{\mathrm{h}}\right)\left[\tau_{\mathrm{h}}+\tau_{\mathrm{a}}-C_{\mathrm{h}}\left(q_{\mathrm{h}}, \dot{q}_{\mathrm{h}}\right)\right] \delta t^{2}
\end{aligned}
$$

Now, the difference between the expected and measured human model joints position is computed (eq. 97.

$$
\begin{aligned}
& q_{\mathrm{h}}(t+\delta t)-\tilde{q}_{\mathrm{h}}(t+\delta t)=q_{\mathrm{h}}(t)+\dot{q}_{\mathrm{h}}(t) \delta t+ \\
& +M_{\mathrm{h}}^{-1}\left(q_{\mathrm{h}}\right)\left[\tau_{\mathrm{a}}-C_{\mathrm{h}}\left(q_{\mathrm{h}}, \dot{q}_{\mathrm{h}}\right)\right] \delta t^{2}-q_{\mathrm{h}}(t)- \\
& -\dot{q}_{\mathrm{h}}(t) \delta t-M_{\mathrm{h}}^{-1}\left(q_{\mathrm{h}}\right)\left[\tau_{\mathrm{h}}+\tau_{\mathrm{a}}-C_{\mathrm{h}}\left(q_{\mathrm{h}}, \dot{q}_{\mathrm{h}}\right)\right] \delta t^{2}
\end{aligned}
$$

Simplifying this equation results in (eq. 10

$$
q_{\mathrm{h}}(t+\delta t)-\tilde{q}_{\mathrm{h}}(t+\delta t)=M_{\mathrm{h}}^{-1}\left(q_{\mathrm{h}}\right) \tau_{\mathrm{h}} \delta t^{2}
$$

which allows us to write an expression for position discrepancy based observer of human contribution to the motion task $\tau_{\mathrm{h}}$ (eq. 11)

$$
\tau_{\mathrm{h}}^{\mathrm{obs}}=\frac{M_{\mathrm{h}}\left(q_{\mathrm{h}}\right)\left[q_{\mathrm{h}}(t+\delta t)-\tilde{q}_{\mathrm{h}}(t+\delta t)\right]}{\delta t^{2}}
$$

The computations involved in the $\tau_{\mathrm{h}}$ observer are presented schematically in Fig. 2

\section{Experience based human contribution prediction}

The position discrepancy based observer for human contribution estimation presented in Sec. IIII-B only produces the estimate $\tau_{\mathrm{h}}^{\mathrm{obs}}$ after the motion is observed, i.e. after the human has actually applied its contribution $\tau_{\mathrm{h}}$. This results in a one time-step behind (lag) estimation. Assuming that between two consecutive time-steps human contribution does not changes significantly, i.e. $\left|\tau_{\mathrm{h}}(t)-\tau_{\mathrm{h}}(t-\delta t)\right|<\epsilon$; for small $\epsilon$, the estimation of human torque contribution is likely to result in an overall good assistance performance.

As a strategy to compensate for the one-step lag in $\tau_{\mathrm{h}}$ observation, we propose to combine the observer with an experience based human contribution prediction. The idea is based on trial-to-trial learning of the human contribution. During the very first assistance trial, as there is no data to learn from yet, we fully rely on the $\tau_{\mathrm{h}}^{\text {obs }}$ for computing $\tau_{\mathrm{a}}^{*}$. After the first trial, the experience gained -namely the observed human contribution and the task error evolution data computed during the assistance trial, can be used to learn the model for predicting human contribution [16]. We suggest task error evolution and assistive force contribution to be the features for learning such a model (eq. 12)

$$
\tau_{\mathrm{h}}^{\text {pred }}=f\left(e, \int e, \dot{e}, \tau_{a}\right)
$$

Thus, the training dataset is of the following structure: feature vector $\left(e(t), \int e(t) d t, \dot{e}(t), \tau_{\mathrm{a}}(t)\right)$; label $\left(\tau_{\mathrm{h}}^{\text {obs }}(t+\delta t)\right)$. 
During the next assistance trial (with the same human subject and for the same task), besides relying only on the observed human contribution $\tau_{\mathrm{h}}^{\text {obs }}$, we can also make use of the ability to predict $\tau_{\mathrm{h}}^{\text {pred }}$ and anticipate what the human contribution is likely to be at the upcoming time-step based on the model learned from previous assistance experiences.

The final $\tau_{\mathrm{h}}$ estimate is then computed as a weighted sum of two terms: observer term and prediction term (eq. 13)

$$
\hat{\tau}_{\mathrm{h}}^{\mathrm{fin}}=(I-W) \tau_{\mathrm{h}}^{\mathrm{obs}}+W \tau_{\mathrm{h}}^{\mathrm{pred}},
$$

where $W$ is the diagonal matrix of prediction confidence weights and $I$ is the identity matrix. $W$ is updated online based on the evaluation of the human contribution prediction model test error. After each time-step, the difference between predicted human contribution and the one observed will inform the system how accurate the experience-based prediction model is (eq. 14).

$$
E_{\text {test }}=\left|\tau_{\mathrm{h}}^{\text {pred }}(t)-\tau_{\mathrm{h}}^{\mathrm{obs}}(t+\delta t)\right| .
$$

If the test error $E_{\text {test }}$ is large for a given human model joint, it is a sign that actual human contribution is significantly different from what was learned from previous experiences (e.g. the human is in a better shape and thus can contribute more than in the previous trials). In this case, the corresponding element on the diagonal of $W$ is decreased. If the prediction matches closely the observed $\tau_{h}^{\text {obs }}$, the $W$ diagonal element is increased. For example, in Sec. IV, the $W$ diagonal element corresponding to the shoulder joint is decreased by a fixed amount of 0.1 until it reaches the value of 0.0 . For increasing this element of $W$ the value of 0.01 is used until the value of the weight reaches 0.9 . With such a strategy, the preference is given to the observer rather than the predictor for the computation of the final estimate of the human torque contribution to the motion task.

After every assistance, the data gathered during the process can be added to the training set and used to retrain and improve the experience based human contribution prediction model. If human performance does not improve or degrade significantly from one trial to another, then with every new assistance trial such a model becomes an increasingly more reliable source of human contribution estimation.

A supplementary benefit of iteratively and continuously training an experience-based human contribution prediction model is the ability to evaluate frailty performance during the assistance trial and report the progress compared to previous assistance experience. If human performance does improve or degrade significantly, this change in human performance can be detected by monitoring the magnitude and sign of the prediction error $E_{\text {test }}$ recorded during the assistance process. For instance, if the observed human contribution is systematically higher than the predicted one, it can be detected using the proposed system and subsequently reported to medical checks.

\section{Force control for human assistance via $M Q P$}

In the previous sections, we explained the strategies for computing $\tau^{\text {task }}$ and estimating $\tau_{\mathrm{h}}$. With these quantities, we can compute the target amount of assistive torque (eq. 15.

$$
\text { if }\left|\hat{\tau}_{\mathrm{h}}^{\mathrm{fin}}\right|<\left|\tau_{\text {task }}\right|: \tau_{\mathrm{a}}^{*}=\tau^{\text {task }}-\hat{\tau}_{\mathrm{h}}^{\text {fin }} \text {; else: } \tau_{\mathrm{a}}^{*}=0
$$

Here, we explain how this value is used in the MQP to compute the amount of required interaction contact force for humanoid-to-human physical assistance.

Both humanoid and personalized human models are included in a single MQP formulation with all related typical MQP constraints and objectives (eq. 16, see details in [6],

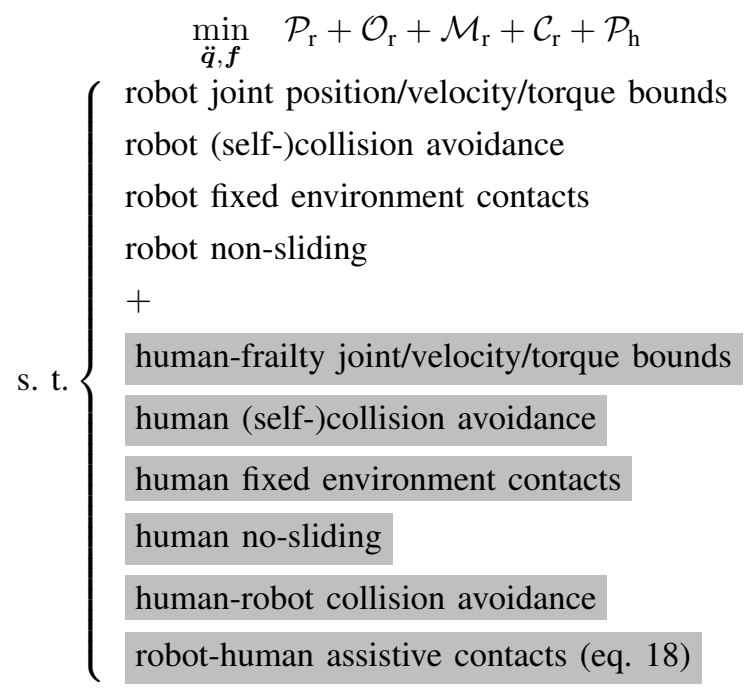

where $\mathcal{P}_{\mathrm{r}}$ and $\mathcal{P}_{\mathrm{h}}$ are robot and human model posture tasks respectively, $\mathcal{O}_{\mathrm{r}}$ is robot head orientation task, $\mathcal{M}_{\mathrm{r}}$ is robot mobile base position task, $\mathcal{C}_{\mathrm{r}}$ is robot CoM task, $\ddot{\boldsymbol{q}}$ and $\boldsymbol{f}$ are MQP decision variables accelerations of the joints of the models and interaction contact forces. A set of contact constraints between two models is defined. The contact point locations are planned w.r.t to the assistance task as in [3].

The feasibility of the physical interaction is ensured by including a combined robot-human equation of motion as a dynamics constraint in MQP, where robot-human interaction forces $F_{\mathrm{rh}}$ are part of the QP decision variables $f$ (eq. 17)

$$
\begin{array}{r}
\left(\begin{array}{cc}
M_{\mathrm{h}}\left(q_{\mathrm{h}}\right) & 0 \\
0 & M_{\mathrm{r}}\left(q_{\mathrm{r}}\right)
\end{array}\right) \ddot{q}+\left(\begin{array}{cc}
C_{\mathrm{h}}\left(q_{\mathrm{h}}, \dot{q}_{\mathrm{h}}\right) & 0 \\
0 & C_{\mathrm{r}}\left(q_{\mathrm{r}}, \dot{q}_{\mathrm{r}}\right)
\end{array}\right)= \\
\quad=S \tau+\left(\begin{array}{cc}
J_{\mathrm{eh}}^{T} & 0 \\
0 & J_{\mathrm{er}}^{T}
\end{array}\right) F_{\mathrm{e}}+\left(\begin{array}{cc}
J_{\mathrm{rh}}^{T} & 0 \\
0 & -J_{\mathrm{hr}}^{T}
\end{array}\right) F_{\mathrm{rh}}
\end{array}
$$

Here $q=\left(q_{\mathrm{h}}, q_{\mathrm{r}}\right), \tau=\left(\tau_{\mathrm{h}}, \tau_{\mathrm{r}}\right)$, where $q_{\mathrm{r}}$ is the vector of robot joint positions and $\tau_{\mathrm{r}}$ is the vector of robot joint torques. $F_{\mathrm{e}}=\left(F_{\mathrm{eh}}, F_{\mathrm{er}}\right)$, and $F_{\mathrm{eh}}, F_{\mathrm{rh}}, F_{\mathrm{er}}$ are stacked vectors of environment-human, robot-human and environment-robot exerted forces respectively. For instance, for $m$ environmenthuman contacts $F_{\mathrm{eh}} \in \mathbb{R}^{6 m}$, with corresponding Jacobians stacked into $J_{\text {eh }} \in \mathbb{R}^{6 m \times d_{\mathrm{h}}}$, where $d_{h}$ is number of DoF in the human body model. The diagonal selection matrix $S$ indicates actuated DoFs, i.e. selection matrix diagonal elements are 1 for the actuated joints and 0 for the DoFs representing the floating bases or none-actuated joints of both humanoid robot and a human model.

Each contact between the humanoid and the human model is represented in the MQP by the following constraint,

$$
J_{\mathrm{rp}} \dot{q}_{\mathrm{r}}=J_{\mathrm{hp}} \dot{q}_{\mathrm{h}}
$$

where $J_{\text {rp }}$ in a robot model link body Jacobian at the contact point $p$ and $J_{\mathrm{hp}}$ is a corresponding body Jacobian at a contact point on a human model, see [6] for more details. 
Having $\tau_{a}^{*}$, we know what assistive torque the robot needs to generate, which can be incorporated into MQP as a constraint $\tau_{\mathrm{a}}^{*}=-J_{\mathrm{hp}}^{T} f_{\mathrm{rh}}$. Yet, considering many other constraints, it might be unfeasible for a robot to fulfill a strict equality constraint. In this case, the target assistive torque can rather be incorporated into MPQ objective function as a task (eq. 19]

$$
\left\|\tau_{\mathrm{a}}^{*}+J_{\mathrm{hp}}^{T} f_{\mathrm{rh}}\right\|
$$

The amount of assistive force is computed by the MQP solver along with desired robot motion that fulfills all MQP constraints. In order for a position-controlled robot to realize the desired assistive force $f_{\mathrm{rh}}$, an admittance task is added into the MQP. This task takes the difference between desired force and the actual one (sensor readings or contact force observer) and outputs the desired end-effector velocity to minimize this difference, see [6].

\section{EXPERIMENTAL RESULTS}

\section{A. Data description}

Due to the pandemic situation, the hardware limitations of Pepper and other practical legal issues (ethical procedures, etc.) it is not yet possible to achieve experiments with real frail patients. In order to assess our approach, we found real patient data that gathers both normal and deficient comparative motions for a given set of simple tasks. We borrow a data sef from a rehabilitation exercise [17] and adapt it to our robotic assistance case-study. The data set gathers recordings of both optimal tasks execution (that we considered to be our reference motion with full muscle strength) as well as nonoptimal motions for similar tasks in case of muscle deficiency (that we consider to emulate human frailty). Note that the reduced version of the dataset [18] is used in our work.

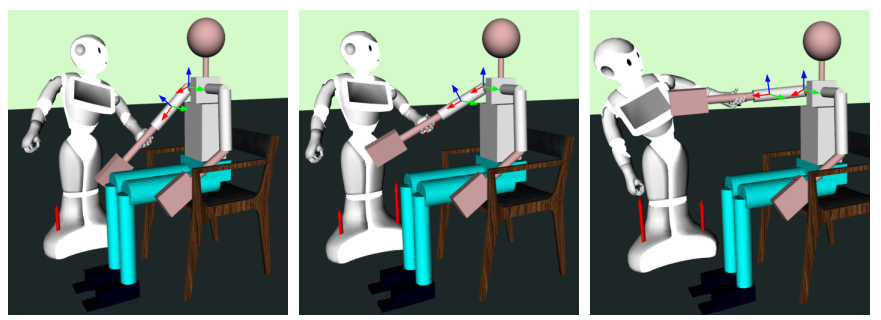

Fig. 3: MQP controller scenes of the interaction wrench and robot motion computation for assistance under pHRI constraints.

We validate our control scheme in simulation (one run) using the human motion recordings of the shoulder scaption rehabilitation exercise. This case is chosen for its achievability by Pepper. According to this task a human is required to raise one arm in front of the chest until reaching the shoulders height, while other joints remain static, see Figs. 1 and 3 . The data set contains 54 repetitions of the shoulder scaption task performed by 6 different subjects ( 9 repetitions per subject).

From the whole-body motion recordings, we extract the right shoulder joint position around the Y-axis (green arrow in Fig. 33; it is the primary joint involved in the scaption

${ }_{1}^{1}$ https://webpages.uidaho.edu/ui-prmd/ task. The other joints of the human model are kept at a fixed position in our study, so the assistance is supplied primarily to the shoulder Y-axis joint. Moreover, the human model is configured to be in a sitting posture to enable Pepper to reach the right forearm and maintain the contact as the human arm is moving upwards during the scaption task.

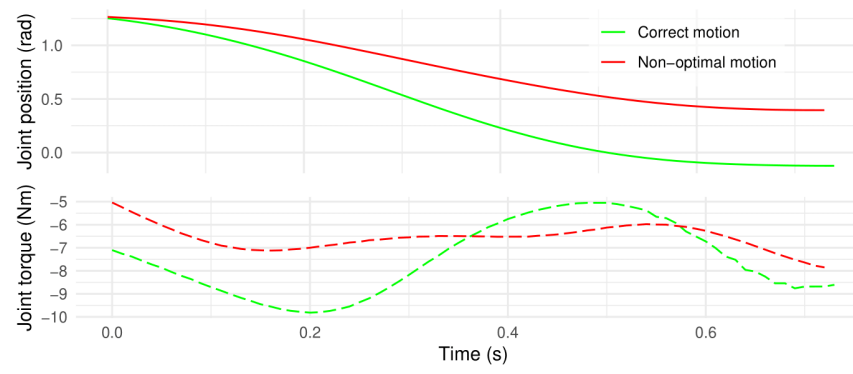

Fig. 4: The correctly performed shoulder scaption (green), non-optimal performance of the same task (red). Position and torque plots for the right shoulder joint around Y-axis.

The top plot in Fig. 4 shows that compared to the correctly performed task, non-optimal (that we consider frail) human motion recording failed to reach the shoulders height level. The bottom plot shows that the range of torques for the frail motion recording is indeed narrower compared to the correct one. Therefore, this sample data is suitable for our study and emulates well the lack of human joint torque (frailty) to achieve the desired scaption task.

\section{B. Computing torques required for the task}

We use a single sample correctly executed scaption task motion recording to identify the task torque control model as described in Sec. III-A Fig. 5 shows the performance of the $\mathrm{NN}$ model in computing task torque from the information of the task error evolution.

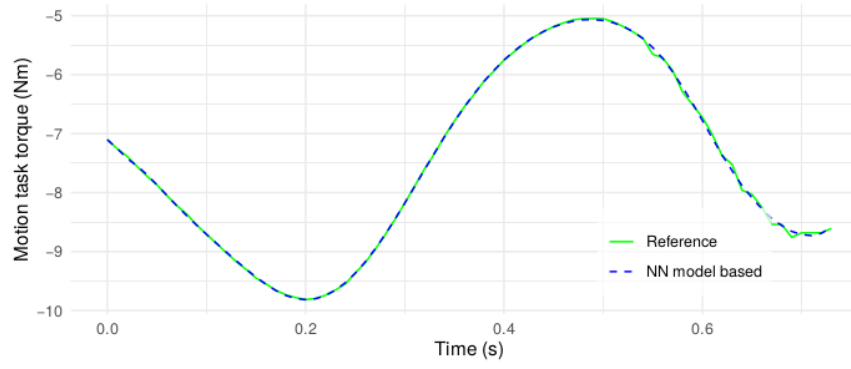

Fig. 5: Identified model of the task torque.

Using the NN model, it is hence possible to compute a task torque that matches closely the reference torque computed from the correct motion recording sample and a personalized human model.

\section{Estimated human contribution}

Now we have in hands the model to compute the $\tau^{\text {task }}$. We assess our proposed human contribution observer $\tau_{\mathrm{h}}^{\text {obs }}$. At this stage, we consider that no previous assistance trials took 
place, therefore no human contribution models exist yet. That is to say: $\left.\tau_{\mathrm{h}}^{\text {pred }}=\mathrm{NA} ; W=\mathbf{0} ; \tau_{\mathrm{h}}^{\mathrm{fin}}=\tau_{\mathrm{h}}^{\mathrm{obs}}\right)$. Fig. 6 shows the observed and true value (unknown to the controller) of the human torque contribution.

Assuming that the position and velocity of the human model joints $\left(q_{\mathrm{h}}(0), \dot{q}_{\mathrm{h}}(0)\right)$ are measured before the start of the assistance process, the model-based value is used as the initial guess of human contribution estimate (eq. 20.

$$
\hat{\tau}_{\mathrm{h}}^{\text {init }}=C_{\mathrm{h}}\left(q_{\mathrm{h}}(0), \dot{q}_{\mathrm{h}}(0)\right)
$$

The observed human contribution data, collected in the previous (first) assistance trial, excluding the initial guess, is used to train the experience-based human contribution prediction model as described in Sec. III-C. Starting from the second assistance trial, this model is used in combination with the observer to improve the final human torque contribution estimate. Fig. 6 shows that the initial guess of the human contribution is improved by $1.76 \mathrm{Nm}$ using the experiencebased prediction model, reducing the estimation error by nearly 10 folds from $1.96 \mathrm{Nm}$ to $0.2 \mathrm{Nm}$. This plot also shows

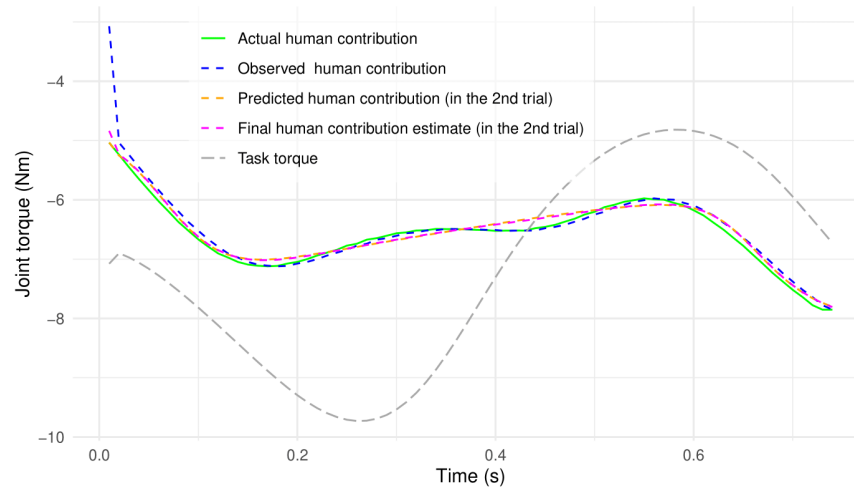

Fig. 6: Estimated human contribution.

that the human torque contribution starts exceeding the task torque after about $0.44 \mathrm{sec}$ of the assistance process. After this point, according to eq. 15, the human contribution to the motion alone is considered to be sufficient to achieve the desired task, the robot is thus moving along with the human, but is not required to generate any assistive force.

\section{Assisted motion}

Having both $\tau^{\text {task }}$ and $\tau_{h}^{\text {fin }}$, the assistive torque $\tau_{\mathrm{a}}$ can be computed (eq. 3. As described in Sec. III-D the human model state and the required amount of assistive torque are integrated into the MQP controller. The latter computes the interaction forces and robot motion to perform the assistance process while satisfying the human model-, robot- and contact constraints and minimizing the MQP tasks erros in the objective function (eq. 16.

The MQP computed robot-to-human assistive force for the first trial (with no prediction of the human contribution) is shown in Fig. 7. The same computation results for the second trial (this time, with the prediction of the human contribution) are shown in Fig. 8. The MQP controller scenes during the force computation process at different times of the scaption task are shown in Fig. 3. In Fig. 6, it is shown how the use of the predictive model helps to reduce the estimation error for the initial guess of the human contribution by nearly 10 folds. This in turn results in lower $\tau_{\mathrm{a}}$ being computed at the very start of the interaction, and consequently lower interaction forces being computed by MQP (Fig. 8). This results in lower (and closer to the reference motion) human joint acceleration at the start of the assistance process (Fig. 10).

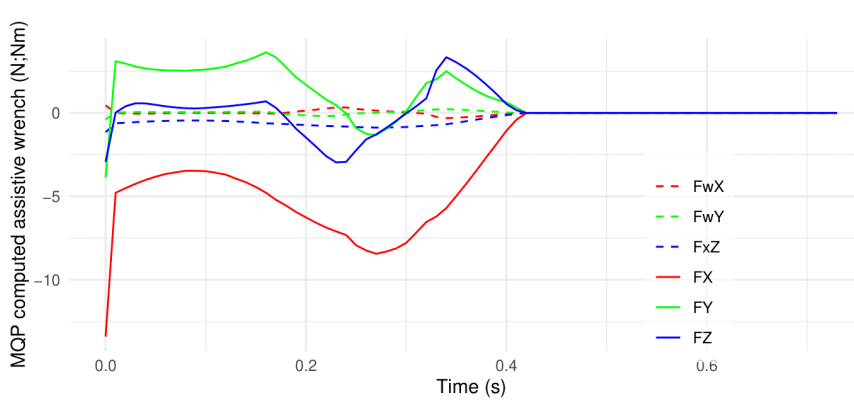

Fig. 7: MQP computed assistive wrench.

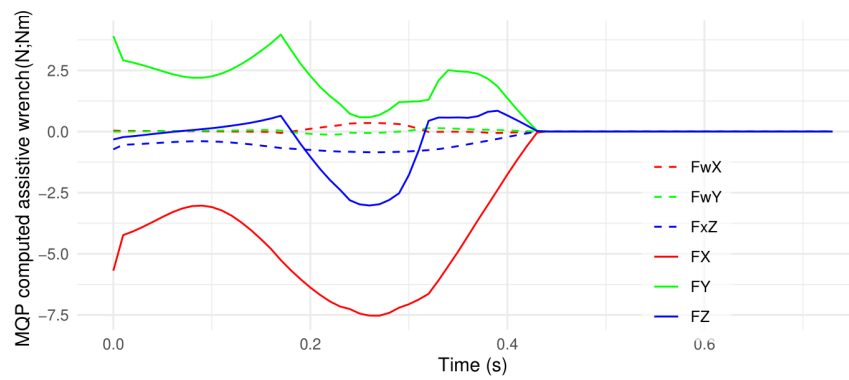

Fig. 8: MQP assistive wrench with experience-based human contribution prediction.

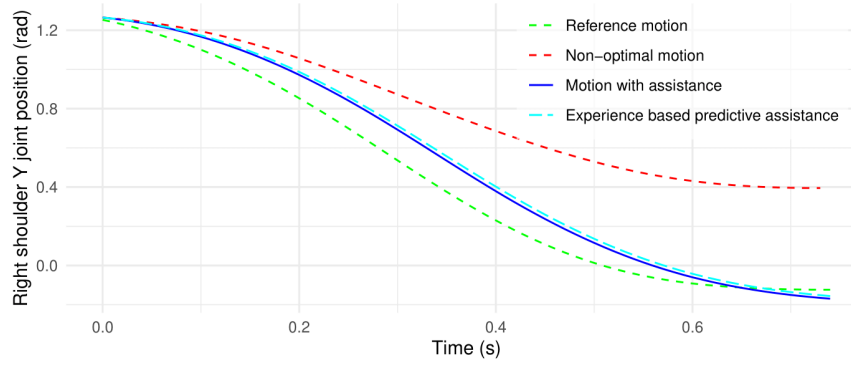

Fig. 9: Joint position during correct motion, non-assisted nonoptimal motion and non-optimal assisted motion.

The MQP computed force is applied to the human model forearm link in PyBullet [19] simulation along with the simulated insufficient human torque contribution, the result is the improved motion performance shown in Figs. 9 and 10 These plots demonstrate that supplied assistive forces help to achieve the scaption task motion that is closer to the correct full muscle strength reference motion.

\section{E. Multi-contact assistance for multi-joint motion}

In order to provide humanoid-to-human assistance in motion for multiple joints, several contacts can be established with 


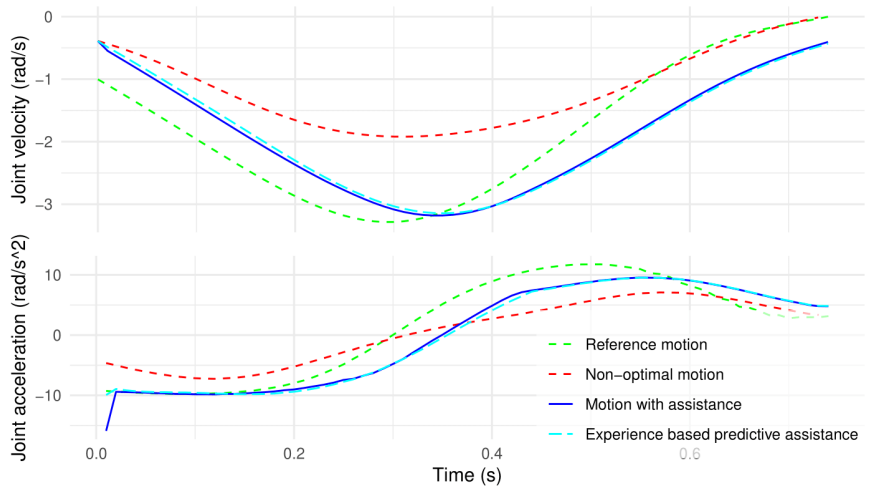

Fig. 10: Joint velocity and acceleration during correct motion, non-assisted non-optimal motion and non-optimal assisted motion.

a human model. For each contact, an assistance requirement is defined as a constraint or a task on the contact force as described in Sec. III-D

Such a multi-contact assistive scenario is represented here by the following example. A contact between the Pepper robot's left end-effector and the human model upper arm is established to supply the required assistive torque at the human model shoulder joint, same as in the simulated experiment presented in the previous subsections. An additional contact, between the robot's right end-effector and the human model hand, is established to generate assistive torque at the human model elbow joint to support elbow bending motion (Fig. 11p. The $\tau_{\text {task }}$ for the bending of the elbow is computed from simulating a human model performing the required motion unassisted. The human model frailty is then simulated by setting $\tau_{a}=0.2 \tau_{\text {task }}$ for the elbow joint.
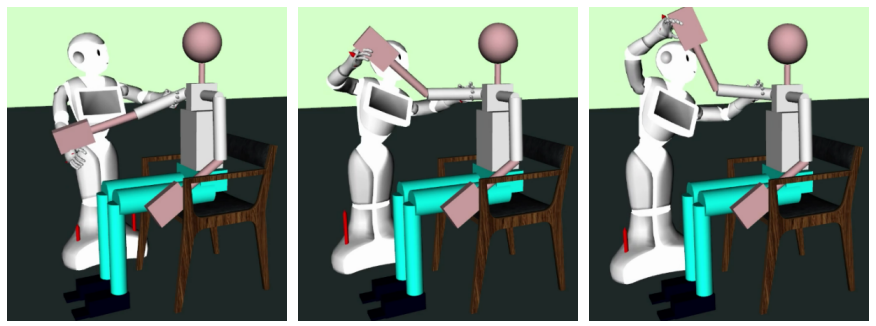

Fig. 11: Humanoid-to-human assistance with two contacts.

\section{F. Discussion, limitations, and future work}

Our proposed method and presented simulation results are based on full knowledge of the frail human model kinematics and dynamic parameters. The human state, namely position and velocity, is also assumed measurable at every time-step. Finally, in the simulation results, it is assumed that the robot can apply the desired assistive forces $f_{\mathrm{rh}}$, as computed by the MQP, to generate the required assistive torque $\tau_{\mathrm{a}}$.

Estimating human model inertia parameters might require an instrumented apparatus, like in [13], [14], or not, like in [20] for the elderly. As the human torque contribution observer and the MQP part of our method rely on human model knowledge, their performance depends on the accuracy of such estimation techniques. Yet, the MQP is in fact a single QP that operates in closed-loop and also in the task-space, which makes it tolerate uncertainties in such parameters [21]. It is more critical to be very precise in the frailty parameters in terms of human joint range and muscle strength limitations (that can be set in a conservative way) than in the exact inertia parameters identification. Other human external contact forces are computed from the MQP, as they are part of the decision variables. Yet, they can be guided from knowledge of the human dynamic parameters estimation and the knowledge of joint accelerations as in [22], [23]. We claim that relying on frail patients physiological data knowledge is feasible because (i) collecting such data is critical in many applications and businesses driven by the Silver Economy boost; and (ii) health and strength conditions of frail patients are monitored through frequent medical checks. Such data can feed robotics and embedded AI algorithms.

An alternative pathway is to make the proposed framework robust to less critical uncertainties by means of domain randomization technique [24], [25]. The proposed method could be extended with an iterative reinforcement learning of the optimal assistive strategy while different variations of the human model are being tested against the method to help with better transfer of the efficient assistive strategy from simulation to real experiments.

In order for the position-controlled Pepper robot to realize the assistive forces, a closed-loop admittance task based system needs to be implemented as part of the interaction MQP controller. This would require a real-time force sensing solution to be implemented on the robot, either as an additional sensor or preferably as a proprioceptive sensor-based estimator [26], [27], [28].

Last, once the method is safely transferred from simulation to real experiments, user studies must be conducted to evaluate how the proposed robotic assistance is perceived by the users and answer such important questions as: (i) does the interaction process feel safe and intuitive?, (ii) does the assistance provided by the robot feel useful?, etc. Additionally, the benefits of using humanoid robot technology in this context can be questioned. Maybe simpler robots specifically designed for a given task is a better solution [29], [30]? Nevertheless, the use of the humanoid technology allows developers to provide a more user-friendly pHRI experience through the use of additional Pepper humanoid features, such as verbal, visual and body language communication [31]. Besides providing physical assistance, a more interactive robot with a humanoid form can do more domestic tasks and provide encouragement via communication channels familiar to the human users [32], [33]. Although the base mobility plays an important role in repositioning, the lack of critical degrees of freedom in the Pepper wrists (one rotation only) is what generated the awkward postures seen in Fig 3. Because of this lack of dexterity, closed-kinematic chain subsequent to both arms contact manipulation of a human (arm) has very limited robot motions as can be seen in Fig 11. Therefore, dexterity and redundancy are important to consider in future versions of Pepper together with grasp/arm strength, to hold human limbs 
during assistance, and a less bulky mobile base to avoid colliding bed and chairs in the vicinity of the human.

\section{CONClusion}

In this letter, we studied humanoid-to-human physical assistance for known task-motions. An adaptive force control framework is proposed for a humanoid to supply the required assistance. The proposed method consists of several interacting components. The first one is the model for computing human joint torque required to achieve the desired task; it is trained on sample motion data. The second component is the observer of the actual and potentially insufficient human contribution to the task. The third component is the experience-based human task contribution model training. The final component includes frailty constraints and consists of the multi-robot whole-body task-space control that computes both the robot motion and the amount of assistive force to apply on the human body to generate the required amount of the assistive torque. We exemplify and discuss the performance of the proposed method on a sample humanoid-to-human assistance using the data of a human subject performing an exercise in a non-optimal way. Limitations and future venues of research and development are also thoroughly discussed.

\section{REFERENCES}

[1] M. Niemelä and H. Melkas, "Robots as social and physical assistants in elderly care," in Human-Centered Digitalization and Services, vol. 19, pp. 177-197, 2019.

[2] T. L. Mitzner, T. L. Chen, C. C. Kemp, and W. A. Rogers, "Identifying the potential for robotics to assist older adults in different living environments," International Journal of Social Robotics, vol. 6, no. 2, pp. 213-227, 2014.

[3] A. Bolotnikova, S. Courtois, and A. Kheddar, "Multi-contact planning on humans for physical assistance by humanoid," in IEEE Robotics and Automation Letters, vol. 5, pp. 135-142, January 2020.

[4] A. Pandey and R. Gelin, "A mass-produced sociable humanoid robot: pepper: the first machine of its kind," IEEE Robotics \& Automation Magazine, vol. 25, no. 3, pp. 40-48, 2018

[5] I. Papadopoulos, C. Koulouglioti, R. Lazzarino, and S. Ali, "Enablers and barriers to the implementation of socially assistive humanoid robots in health and social care: a systematic review," BMJ open, vol. 10, no. 1, 2020.

[6] K. Bouyarmane, K. Chappellet, J. Vaillant, and A. Kheddar, "Quadratic programming for multirobot and task-space force control," IEEE Transactions on Robotics, vol. 35, no. 1, pp. 64-77, 2019.

[7] E. Todorov and M. I. Jordan, "Optimal feedback control as a theory of motor coordination," Nature Neuroscience, vol. 5, no. 11, pp. 1226$1235,2002$.

[8] P. Kratzer, M. Toussaint, and J. Mainprice, "Towards combining motion optimization and data driven dynamical models for human motion prediction," in IEEE-RAS International Conference on Humanoid Robots, pp. 202-208, 2018.

[9] R. Aissaoui and J. Dansereau, "Biomechanical analysis and modelling of sit to stand task: a literature review," in IEEE International Conference on Systems, Man, and Cybernetics, vol. 1, pp. 141-146, 1999.

[10] P. Kratzer, M. Toussaint, and J. Mainprice, "Prediction of human fullbody movements with motion optimization and recurrent neural networks," in IEEE International Conference on Robotics and Automation, pp. 1792-1798, 2020.

[11] E. Corona, A. Pumarola, and G. A. F. Moreno-Noguer, "Contextaware human motion prediction," in IEEE/CVF Conference on Computer Vision and Pattern Recognition, pp. 6990-6999, 2020.

[12] I. Herman, Physics of the human body. Springer Science \& Business Media, 2007.

[13] J. Jovic, A. Escande, K. Ayusawa, E. Yoshida, A. Kheddar, and G. Venture, "Humanoid and human inertia parameter identification using hierarchical optimization," IEEE Transactions on Robotics, vol. 32, pp. 726-735, June 2016.
[14] C. Latella, M. Lorenzini, M. Lazzaroni, F. Romano, S. Traversaro, M. A. Akhras, D. Pucci, and F. Nori, "Towards real-time whole-body human dynamics estimation through probabilistic sensor fusion algorithms," Autonomous Robots, vol. 43, no. 6, pp. 1591-1603, 2019.

[15] Z. Cao, G. Hidalgo, T. Simon, S.-E. Wei, and Y. Sheikh, "OpenPose: realtime multi-person 2D pose estimation using part affinity fields," IEEE Transactions on Pattern Analysis and Machine Intelligence, vol. 43, no. 1 , pp. 172-186, 2021.

[16] E. Gribovskaya, A. Kheddar, and A. Billard, "Motion learning and adaptive impedance for robot control during physical interaction with humans," in IEEE International Conference on Robotics and Automation, pp. 4326-4332, May 2011.

[17] A. Vakanski, H.-p. Jun, D. Paul, and R. Baker, "A data set of human body movements for physical rehabilitation exercises," Data, vol. 3, no. 1, p. 2, 2018.

[18] Y. Liao, A. Vakanski, and M. Xian, "A deep learning framework for assessing physical rehabilitation exercises," IEEE Transactions on Neural Systems and Rehabilitation Engineering, vol. 28, no. 2, pp. 468477, 2020.

[19] E. Coumans and Y. Bai, "Pybullet, a python module for physics simulation for games, robotics and machine learning." http://pybullet.org 2016-2019.

[20] M. J. Pavol, T. M. Owings, and M. D. Grabiner, "Body segment inertial parameter estimation for the general population of older adults," Journal of biomechanics, vol. 35, no. 5, pp. 707-712, 2002.

[21] K. Bouyarmane and A. Kheddar, "On weight-prioritized multitask control of humanoid robots," IEEE Transactions on Automatic Control, vol. 63, no. 6, pp. 1632-1647, 2018.

[22] T.-H. Pham, N. Kyriazis, A. A. Argyros, and A. Kheddar, "Handobject contact force estimation from markerless visual tracking," IEEE Transactions on Pattern Analysis and Machine Intelligence, vol. 40, no. 12 , pp. $2883-2896,2018$.

[23] T.-H. Pham, S. Caron, and A. Kheddar, "Multicontact interaction force sensing from whole-body motion capture," IEEE Transactions on Industrial Informatics, vol. 14, no. 6, pp. 2343-2352, 2018.

[24] J. Tobin, R. Fong, A. Ray, J. Schneider, W. Zaremba, and P. Abbeel, "Domain randomization for transferring deep neural networks from simulation to the real world," in IEEE/RSJ International Conference on Intelligent Robots and Systems, pp. 23-30, 2017.

[25] J. Tremblay, A. Prakash, D. Acuna, M. Brophy, V. Jampani, C. Anil, T. To, E. Cameracci, S. Boochoon, and S. Birchfield, "Training deep networks with synthetic data: Bridging the reality gap by domain randomization," in IEEE Conference on Computer Vision and Pattern Recognition Workshops, pp. 969-977, 2018.

[26] G. Buondonno and A. De Luca, "Combining real and virtual sensors for measuring interaction forces and moments acting on a robot," in IEEE/RSJ International Conference on Intelligent Robots and Systems, pp. 794-800, 2016.

[27] F. Flacco and A. Kheddar, "Contact detection and physical interaction for low cost personal robots," in IEEE International Symposium on Robot and Human Interactive Communication, pp. 495-501, 2017.

[28] S. A. B. Birjandi, J. Kühn, and S. Haddadin, "Observer-extended direct method for collision monitoring in robot manipulators using proprioception and imu sensing," IEEE Robotics and Automation Letters, vol. 5, no. 2, pp. 954-961, 2020.

[29] X. Li, Y. Pan, G. Chen, and H. Yu, "Adaptive human-robot interaction control for robots driven by series elastic actuators," IEEE Transactions on Robotics, vol. 33, no. 1, pp. 169-182, 2016.

[30] C. Lee, J.-Y. Kim, S.-Y. Kim, and S. Oh, "Human force observation and assistance for lower limb rehabilitation using wire-driven series elastic actuator," Mechatronics, vol. 55, pp. 13-26, 2018.

[31] A. Bolotnikova, S. Courtois, and A. Kheddar, "Autonomous initiation of human physical assistance by a humanoid," in IEEE International Conference on Robot and Human Interactive Communication, (Napoli, Italy), 31 August - 4 September 2020.

[32] D. Li, P. P. Rau, and Y. Li, "A cross-cultural study: Effect of robot appearance and task," International Journal of Social Robotics, vol. 2, no. 2, pp. 175-186, 2010.

[33] E. Torta, F. Werner, D. O. Johnson, J. F. Juola, R. H. Cuijpers, M. Bazzani, J. Oberzaucher, J. Lemberger, H. Lewy, and J. Bregman, "Evaluation of a small socially-assistive humanoid robot in intelligent homes for the care of the elderly," Journal of Intelligent \& Robotic Systems, vol. 76, no. 1, pp. 57-71, 2014. 